\title{
Comunicação de notícias difíceis na atenção básica à saúde: percepção dos estudantes de enfermagem
}

\author{
Communicating difficult news in primary health care: Nursing students' perceptions \\ Comunicación de noticias difíciles en la atención de salud básica: percepción de estudiantes de enfermería
}

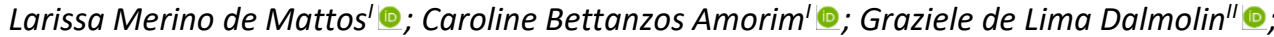 \\ Rafael Chiesa Avancini' ${ }^{\circledR}$; Felipe Godinho Lima ${ }^{\prime \prime \prime}{ }^{\circledR}$; Edison Luiz Devos Barlem' ${ }^{\circledR}$
}

'Universidade Federal do Rio Grande, Rio Grande, RS, Brasil; "Universidade Federal de Santa Maria, Santa Maria, RS, Brasil; I'Unicesumar, Rio Grande, RS, Brasil

\begin{abstract}
RESUMO
Objetivo: conhecer a percepção dos estudantes de enfermagem acerca da comunicação de notícias difíceis na Atenção Básica à Saúde mediante suas vivências no período de formação. Método: estudo descritivo com abordagem qualitativa no qual participaram 12 estudantes de enfermagem de uma universidade pública do sul do Brasil. Os dados foram coletados em junho de 2019, a partir da técnica de grupo focal e submetidos à análise textual discursiva. Resultados: emergiram duas categorias: "percepção dos estudantes acerca das notícias difíceis na Atenção Básica à Saúde" e "formação em enfermagem para comunicação de notícias difíceis". Conclusão: percebe-se que os estudantes possuem fragilidades em comunicar notícias difíceis na atenção básica à saúde durante o período de formação. Para que seja possível preencher essa lacuna, as instituições formadoras têm um grande caminho a percorrer, adotando estratégias para o fortalecimento do processo de comunicação de notícias difíceis tanto na teoria quanto na prática.
\end{abstract}

Descritores: Enfermagem; Atenção Primária à Saúde; Comunicação em Saúde; Estudantes.

\begin{abstract}
Objective: to learn Nursing students' perceptions as regards communicating difficult news in Primary Health Care, as experienced during the training period. Method: in this qualitative, descriptive study, the participants were 12 Nursing students from a public university in southern Brazil. Data were collected in June 2019, using the focus group technique, and submitted to discursive textual analysis. Results: two categories emerged: "students' perception of difficult news in Primary Health Care" and "Nursing training to communicate difficult news". Conclusion: the students were found to suffer from weaknesses in communicating difficult news in Primary Health Care during the training period. Training institutions have a long way to go to fill this gap by adopting strategies to strengthen the process of communicating difficult news in both theory and practice. Descriptors: Nursing; Primary Health Care; Health Communication; Students.
\end{abstract}

\section{RESUMEN}

Objetivo: conocer la percepción de los estudiantes de enfermería sobre la comunicación de noticias difíciles en la Atención Primaria de Salud a través de sus experiencias durante el período de formación. Método: estudio descriptivo con enfoque cualitativo en el que participaron 12 estudiantes de enfermería de una universidad pública del sur de Brasil. Los datos fueron recolectados en junio de 2019, con base en la técnica del grupo focal y sometidos al Análisis Textual Discursivo. Resultados: surgieron dos categorías: "percepción de los estudiantes sobre las noticias difíciles en Atención Primaria de Salud" y "capacitación en enfermería para comunicar noticias difíciles". Conclusión: se observa que los estudiantes tienen debilidades para comunicar noticias difíciles en la atención primaria de salud durante el período de formación. Para poder llenar este vacío, las instituciones a cargo de la capacitación tienen un largo camino por recorrer, adoptando estrategias para fortalecer el proceso de comunicación de noticias difíciles, tanto en la teoría como en la práctica.

Descriptores: Enfermería; Atención Primaria de Salud; Comunicación en Salud; Estudiantes.

\section{INTRODUÇÃO}

A comunicação na assistência à saúde na Atenção Básica é recurso indispensável e essencial nas relações humanas, seja com a equipe ou com usuários e familiares, pois além de ser fundamental para que se realize uma assistência efetiva e de qualidade, está intimamente ligada ao processo de humanização ${ }^{1,2}$.

Percebe-se que na Atenção Básica à Saúde são verificadas situações que exigem a comunicação de notícias difíceis aos usuários, no entanto, as mesmas muitas vezes não são reconhecidas como parte de um processo de comunicação de uma má notícia. Essas possuem relação direta com os procedimentos realizados pela enfermagem, como por exemplo, na realização de testes rápidos (HIV; Sífilis e Hepatites); no diagnóstico de gravidez; na consulta de enfermagem (coleta de citopatológico); no acompanhamento de pacientes crônicos, o que as torna um problema potencial tanto para quem transmite a mensagem, quanto para quem recebe.

Agradecimentos ao apoio da Coordenação de Aperfeiçoamento de Nível Superior - Brasil (CAPES) pelo fomento ao Programa de Pós-Graduação em Enfermagem da Universidade Federal do Rio Grande.

Autora correspondente: Larissa Merino de Mattos, merinolarissa@hotmail.com

Editora Científica: Cristiane Helena Gallasch; Editora Associada: Sonia Acioli Oliveira 
Notícias difíceis são consideradas aquelas que modificam drástica e negativamente a visão de presente/futuro do paciente. Geralmente, são relacionadas a situações que representam ameaça à vida, ao bem-estar pessoal, familiar e social, além das consequências físicas e psicossociais que podem ocasionar aos indivíduos ${ }^{3}$. Estar envolvido no processo de comunicar notícias difíceis é uma tarefa complexa que exige conhecimento e habilidade, pois uma comunicação bem realizada poderá trazer benefícios aos usuários, ajudando-os a suportar as consequências da notícia, assim como uma comunicação ineficaz pode ter efeitos drásticos e duradouros ${ }^{4}$, portanto, é importante que a comunicação de notícias difíceis seja aperfeiçoada pelos profissionais que transmitem a informação e compreendida pelo indivíduo que deve receber a notícia, de maneira que faça-o entender a gravidade de forma clara.

Diante do exposto, o estudo teve como objetivo conhecer a percepção dos estudantes de enfermagem acerca da comunicação de notícias difíceis na Atenção Básica à Saúde mediante suas vivências no período de formação. Configurou-se como problema de pesquisa o desconhecimento sobre a percepção dos estudantes de enfermagem em suas vivências acadêmicas e estágios obrigatórios da graduação, sobre como lidar com a comunicação de notícias difíceis na Atenção Básica à Saúde.

Alicerçada nas produções científicas, esta pesquisa justificou-se a partir dos seguintes pontos: a relevância da discussão acadêmica sobre como enfrentar a situação de comunicar notícias difíceis aos usuários na Atenção Básica à Saúde; a escassez na produção científica sobre o tema ${ }^{4}$; a necessidade de produção de conhecimento que contribua para a prática cotidiana dos futuros profissionais, devido à temática não estar presente em grande parte dos currículos dos cursos de graduação da área da saúde.

\section{MÉTODO}

Trata-se de um estudo descritivo com abordagem qualitativa. Participaram do estudo 12 estudantes do curso de graduação em enfermagem do sétimo ao décimo semestre de uma universidade Federal do Sul do Brasil. Justificou-se a escolha dos participantes pelo fato de estarem cursando as atividades práticas na rede de atenção básica. Para seleção dos participantes, foi utilizada a amostragem não probabilística do tipo bola-de-neve ${ }^{5}$, onde o primeiro participante selecionado, escolhido previamente pelo pesquisador, indicou o próximo e assim consecutivamente, até atingir um total de 12 estudantes. O primeiro participante foi escolhido por ser reconhecido com um estudante que previamente havia manifestado a importância da temática, além de ter reconhecido suas limitações durante a realização das atividades práticas durante as disciplinas do curso de graduação.

Os critérios de inclusão foram: ser estudante regular do curso de graduação em enfermagem cenário do estudo; estar cursando disciplinas práticas de rede básica; aceitar participar do grupo focal após explicação do pesquisador sobre como se daria os encontros; já possuir vivência de comunicar notícias difíceis na Atenção Básica. Os critérios de exclusão foram: estudantes que não estivessem cursando os semestres indicados; relatar não ter vivenciado situações de comunicar uma notícia difícil durante as atividades práticas na atenção básica a saúde.

A pesquisa iniciou após a aprovação do Comitê de Ética local sob o parecer $N^{\circ} 118 / 2019$. Todos os preceitos com relação à ética em pesquisa com seres humanos foram respeitados. O anonimato foi garantido por meio da codificação dos participantes pela letra "E" seguido de um número arábico, em ordem crescente, correspondente à ordem das falas dos participantes durante a realização do grupo focal, "E1, E2..." até chegar ao número total de participantes.

A coleta de dados ocorreu no mês de junho de 2019, através da técnica do Grupo Focal (GF), a qual promove ampla e horizontalizada problematização da temática ${ }^{6}$. Realizaram-se três encontros, nos quais todos os 12 estudantes estiveram presentes. Cada encontro prolongou-se por, no máximo, uma hora e meia e foi coordenado pelo moderador e acompanhado por um observador.

O moderador instigava com perguntas abertas e fechadas até o esgotamento da temática e o observador realizava anotações sobre qual participante estava realizando a fala, percebendo emoções, frustações e fragilidades. 0 encontro foi gravado com aparelho de áudio eletrônico para possível transcrição das falas, com o consentimento de todos os participantes do estudo. Os encontros obtiveram intervalos de uma semana entre eles.

No primeiro encontro foi realizada uma breve apresentação do moderador e do observador, relembrando e explicando como se dariam os encontros e os objetivos do grupo focal. A partir de então, foi realizada uma introdução expositiva do tema e após apresentado o vídeo "Empathy - Cleveland Clinic" com o intuito de observar a compreensão, o conhecimento e as atitudes dos participantes em relação à temática.

Logo após foram entregues folhas em branco com a expressão "Notícias Difíceis", na qual os participantes relataram todos os seus sentimentos com relação à temática e ao vídeo. Por fim, foi solicitado que os estudantes verbalizassem esses sentimentos no intuito de auxiliar na interação, incentivando desse modo o diálogo e a discussão entre o grupo, gerando a saturação do assunto abordando cada uma das palavras e sentimentos descritos pelos participantes. A saturação obteve-se quando os participantes do estudo se tornavam repetitivos. 
O segundo encontro iniciou com a apresentação de dois vídeos, "50/50: El Diagnóstico" e "Comunicando notícias difíceis" o qual mostrou situações de comunicação de notícias difíceis, com vistas a fomentar as discussões com base no que foi visto e propor que os participantes pensassem e refletissem acerca da postura do profissional e dos principais desafios frente à participação do enfermeiro na comunicação de notícias difíceis na Atenção Básica à Saúde. Após, houve discussão acerca da temática abordada até a saturação das falas.

No terceiro e último encontro, foi apresentada uma síntese do segundo encontro, a fim de relembrar as discussões levantadas. Dessa maneira, teve-se como objetivo proposto que o grupo discutisse e apresentasse diferentes e diversas maneiras de como comunicar uma notícia difícil para o usuário e como fazê-lo entender o que estava sendo dito. Assim, tendo o propósito de elaborar e visar novas estratégias que facilitem e tornem eficaz essa comunicação desde o período de formação acadêmica, para que na prática profissional a temática já tivesse sido abordada. Sendo assim, a reunião deu-se até a saturação dos dados conforme os dois primeiros encontros realizados.

Após o encerramento do último encontro, os participantes fizeram um fechamento com um feedback sobre as reuniões onde evidenciaram que a temática é de extrema importância, visto que na graduação, não possuem disciplinas aptas para lidar com a transmissão de notícias difíceis.

O conteúdo do GF foi transcrito e submetido à Análise Textual Discursiva, o qual é baseado em quatro elementos: unitarização, categorização, captação do novo emergente e processo auto-organizado. O primeiro elemento constituiuse pela desconstrução dos textos, objetivando avaliar os materiais em seus detalhes, visando à elaboração de um significado mais completo e à percepção de diferentes sentidos do próprio texto. O segundo elemento, a categorização, foi responsável por unir as partes que foram inicialmente descontruídas e leva ao agrupamento de elementos com significados parecidos. Além disso, buscou nomear e definir as categorias obtidas ${ }^{7}$.

A captação do novo emergente, o terceiro elemento, buscou descrever e interpretar os sentidos comuns do que foi produzido ao longo da pesquisa e tornou compreensível o entendimento da mesma para os leitores. A partir desses três elementos citados, foi possível chegar ao quarto e último elemento, que é denominado processo auto-organizado, pois este foi capaz de criar e recriar uma nova compreensão da pesquisa após a desordem que foi feita durante todo o processo ${ }^{7}$.

\section{RESULTADOS}

Em relação às características dos 12 participantes do curso de graduação em enfermagem, um era do sexo masculino e onze do sexo feminino. Desses, seis estudantes estavam cursando o sétimo semestre, dois o oitavo semestre, um o nono semestre e três o décimo semestre. Sobre a idade, obteve-se uma média aritmética simples de 25 anos. A maioria dos estudantes eram solteiros e não possuíam filhos.

Os resultados desse estudo foram organizados por meio de duas categorias: percepção dos estudantes acerca das notícias difíceis na Atenção Básica à Saúde; formação em enfermagem para a comunicação de notícias difíceis.

\section{Percepção dos estudantes acerca das notícias difíceis na Atenção Básica à Saúde}

Foi possível observar que os estudantes de enfermagem reconhecem uma notável diferença no teor de fornecer notícias difíceis se comparadas à rede básica e hospitalar, realizando o relato das notícias mais recorrentes no cenário da Atenção Básica. Quando instigados a refletirem acerca do termo "notícias difíceis", os estudantes de enfermagem relataram que o termo os remete a casos mais graves, possivelmente envolvendo a possibilidade de morte associada mais ao ambiente hospitalar do que à rede básica.

[...] No hospital as notícias parecem que são mais pesadas. Eu acho que em função das patologias, porque dentro da rede básica a gente trabalha com patologias distintas. (E6)

[...] Na Atenção Básica não vamos ver um número de mortes tão grande quanto no ambiente hospitalar, talvez para nós seja mais cansativo lidar emocionalmente com as notícias que devemos dar aos pacientes devido à questão do vínculo e conhecer o histórico daquela pessoa, daquela família, e isso acaba com o psicológico. (E2)

Na Atenção Básica à Saúde as notícias difíceis que surgem possuem relação com os procedimentos realizados pela enfermagem, como por exemplo, a realização de testes rápidos e a consulta de enfermagem.

Eu tive oportunidade agora na rede básica no primeiro estágio de comunicar notícias difíceis, como, por exemplo, HIV, sifilis e gravidez. E uma notícia difícil, como o HPV NIC II, III é difícil de dar também, porque o papanicolau quem faz e recebe os resultados são os enfermeiros. (E5)

A minha maior dificuldade foi lidar com o lado psicológico da paciente quando eu disse que ela estava grávida, eu não esperava aquela reação, aquele choro, aquele desespero. (E1)

Os estudantes possuem a percepção que a notícia comunicada pelos médicos aos usuários é apenas comunicada, ou seja, não oferece continuidade da assistência com explicações, deixando essa atribuição para o enfermeiro. Com isso, visualizam que, quem fornece o suporte para o usuário após uma notícia difícil é o enfermeiro. 
[...] Eles apresentam a notícia e vão embora e quem acaba tendo que abraçar a causa é a enfermagem. Quantas vezes, numa simples consulta o paciente sai do consultório sem entender nada do que o médico disse. E ele vai perguntar pra quem? Para a enfermeira. (E6)

[...] Só quem fica com o pós da má notícia é sempre nós futuros enfermeiras e enfermeiros, porque quando os pacientes precisarem de informação vai ser para nós que irão se direcionar, quando eles forem procurar porque o exame voltou e não deu certo, vai ser para nós [...]. (E1)

$\mathrm{Na}$ visão dos estudantes, alguns profissionais da saúde, esbarram no ato de postergar as comunicações para o usuário e familiar, buscando evitar tal situação. Na Atenção Básica à Saúde isso pode acontecer devido ao vínculo e a confiança que é estabelecida junto ao usuário/família, sendo assim, os profissionais podem ficar apreensivos de desestruturar essa relação a partir de uma notícia difícil.

[...] Acho que por isso também é tão difícil a gente enquanto futuros enfermeiros comunicar a notícia difícil, porque não está no nosso código de ética, teoricamente não é obrigação nossa. (E4)

Eu, por exemplo, trabalho na unidade e tenho diversas amizades que fiz lá e parece que quanto mais amizade tu tens com a pessoa, mais difícil é de comunicar uma notícia difícil, acho que seria necessária uma equipe multiprofissional para dar suporte. (E6)

\section{Formação em enfermagem para a comunicação de notícias difíceis}

Nesta categoria são elencados os aspectos pontuados pelos estudantes em relação ao modo como a temática da comunicação de notícias difíceis é explorada durante a graduação, o que aponta para importantes fragilidades: falta de abordagem na graduação; carga horária deficiente e falta de estrutura do currículo no processo de comunicação. Consoante com a ótica dos estudantes de enfermagem, a falta de abordagem na graduação foi destacada como fator negativo, pois essa falta de abordagem talvez ocorra porque as atividades desenvolvidas pelo curso de enfermagem possuem ênfase maior no ambiente hospitalar, o que acaba fragilizando a atuação dos estudantes no contexto da Atenção Básica à Saúde.

[...] Eu estava percebendo diversas situações ao longo da graduação que a gente passa que eu acabei fornecendo ou participando de notícias difíceis sem perceber, sem ter tempo de ter uma reação, porque realmente é algo que não é abordado durante a graduação. (E7)

A graduação ela é hospitalar, a graduação não te prepara para a rede básica. A graduação te forma para o ambiente hospitalar [...]. (E8)

Eu nunca passei por uma situação dessas na rede básica, acredito que seja necessária uma maior estrutura do que se fosse no ambiente hospitalar. (E3)

Foram evidenciadas também, fragilidades no quesito carga horária deficiente e falta de estrutura do currículo, uma vez que os estudantes acreditam que o tempo de atuação proposto para a realização de atividades práticas obrigatórias na Atenção Básica à Saúde é muito menor do que o despendido para as atividades práticas hospitalares, dificultando vivenciar as situações recorrentes na Atenção Básica à Saúde.

A gente vai para dentro de uma unidade de saúde só no sétimo semestre. Eu acho que essa é a maior falha. $E$ acho que não tem como aprender a dar uma notícia difícil se a gente não está vivendo aquilo ou nunca vivenciou. (E11)

Desde o início da graduação a gente encontra todos os problemas, a falta de estrutura do nosso currículo é o principal deles em relação a esse assunto. (E1)

A carga horária na rede básica é muito pequena, acredito que precise ser revista essa questão pela coordenação e direção do curso, para melhor consolidar a nossa formação. (E4)

\section{DISCUSSÃo}

Foi possível perceber que os estudantes de enfermagem associam as notícias difíceis a casos mais graves, envolvendo a possibilidade de morte associada mais ao ambiente hospitalar do que a rede básica. Tal achado corrobora com os estudos ${ }^{8,9}$ realizados com profissionais da saúde de uma unidade de terapia intensiva neonatal e pediátrica que constatou que o principal significado associado ao conceito de "más notícias em saúde" é a morte.

Partindo do pressuposto que é dever dos médicos revelar a notícia difícil, ressalta-se que os enfermeiros possuem um fazer importante no fornecimento de notícias aos usuários e familiares, de tal forma que habilidades de comunicação são saberes necessário a serem adquiridos na formação desses profissionais ${ }^{10,11}$. Os enfermeiros são essenciais no processo de comunicação de notícias difíceis, pois ajudam o usuário a compreender e a suportar as informações que receberam ${ }^{12}$. Além disso, possuem a responsabilidade de acompanhar e dar suporte ao usuário após a comunicação de notícias difíceis, o que pode ser justificado por ser o profissional que está em contato diário e direto com usuários e familiares, pois é quem fornece e planeja o cuidado ${ }^{10}$. 
De acordo com a percepção dos estudantes, alguns profissionais da saúde, esbarram no ato de postergar as comunicações para o usuário e familiar, buscando evitar tal situação. No contexto da Atenção Básica à Saúde, essa situação pode ser ainda mais elevada, pois existe a relação baseada no vínculo que, diante de uma notícia difícil, pode ser rompida ${ }^{13}$. O vínculo é elemento indispensável para a consolidação das relações na Atenção Básica à Saúde, é considerado recurso terapêutico e ferramenta primordial para o funcionamento da Atenção Básica à Saúde, uma vez que faz a intermediação da comunicação entre profissionais e usuários concretizando o estabelecimento de uma relação sólida entre eles ${ }^{12,14}$.

A partir do supracitado, pode-se citar o acróstico TERNA, originalmente do inglês - nurse, a mnemônica auxilia os profissionais a reconhecer diferentes emoções do paciente para um melhor cuidado, tais como: reconhecer situações de cunho emocional através de sinais verbais e não verbais emitidos pelo paciente, procurar entender, ser empático frente às situações em que o paciente se encontra, respeitar e não julgar as manifestações emocionais, instigar e apoiar o paciente a conversar e deixá-lo à vontade para se expressar da forma que achar adequado no momento em que vivencia situações difíceis ${ }^{15}$.

Outro importante ponto acerca da temática, diz respeito ao protocolo Spikes, mundialmente conhecido e recomendado a sua utilização para os profissionais para que o instrumento ajude a lidar com conteúdos emocionais e comunicação de más notícias. Seis passos compõem o protocolo de Spikes para dar uma notícia difícil ao usuário, são eles: planejar a entrevista atentamente, avaliar o seu entendimento e compreensão, obter e respeitar a aceitação ou não do paciente do convite para discutir sobre a situação, fornecendo conhecimento e informações ao paciente certificando-se que ele entenda o curso da conversa, atentar as emoções transmitidas e abordá-las com empatia e por fim, elaborar estratégias para lidar com a comunicação de más notícias ${ }^{15,16,17}$.

Dessa maneira, a falta de abordagem na graduação foi percebida pelos estudantes como um fator limitante para a comunicação de notícias difíceis. Um estudo ${ }^{8}$ que pesquisou sobre a formação voltada para a prática de comunicar notícias difíceis verificou que existem falhas nas graduações e que essas variam entre a total falta de preparo acadêmico para enfrentar a situação e a formação inicial mal estabelecida no fluxo do curso, que não qualifica o futuro profissional para comunicar notícias difíceis com qualidade para si e usuários ${ }^{18,19}$

Pondera-se que no Brasil há uma possível fragilidade na formação de profissionais da saúde em relação a assuntos de morte e morrer. Para solucioná-la, é preciso que o tema seja incorporado nas instituições de ensino, assim como em programas de educação continuada para que conduza adequadamente os profissionais às situações que dizem respeito à morte e às notícias difíceis ${ }^{2}$. A inserção da temática nos currículos de graduação pode influenciar positivamente a atitude de futuros profissionais, potencializando as habilidades de comunicação e reduzindo os fatores prejudicais ${ }^{11}$.

Essa inserção pode ser realizada através da inclusão de disciplinas obrigatórias, que contemplem a comunicação de notícias difíceis, na grade curricular dos cursos de graduação em enfermagem, assim como aumentar a carga horária de atividades práticas na Atenção Básica à Saúde, exercitando a tomada de iniciativa acerca da comunicação entre profissional e paciente, visando possibilitar que os estudantes vivenciem as situações mais recorrentes nesse nível de atenção à saúde.

Nesse sentido, é imperativo abrir novos espaços e implantar estratégias educativas para que os futuros profissionais da enfermagem possam refletir sobre esse tema complexo, pois o cenário atual sobre o processo de comunicação de notícias difíceis revela um longo caminho a ser percorrido pelas instituições de ensino para garantir o reconhecimento e a relevância do tema ${ }^{11}$. Os estudantes identificaram fragilidades no quesito carga horária deficiente e falta de estrutura do currículo em relação às atividades desenvolvidas na Atenção Básica à Saúde o que acaba causando um déficit na formação acadêmica. Entretanto, os estudantes nem sempre se sentem preparados para enfrentar esse tipo de situação, o que evidencia ainda mais a necessidade de melhorar o processo de comunicação de más notícias ${ }^{20}$.

\section{Limitações do estudo}

O estudo obteve como limitações uma população específica, sugere-se a realização de outros estudos que corroborem para o aprofundamento do conhecimento acerca da comunicação de notícias difíceis no cenário da Atenção Básica à Saúde. Assim como, a exploração e novas implementação de estratégias para o desenvolvimento da temática durante o período de formação, com foco na Atenção Básica à Saúde.

\section{CONCLUSÃO}

Tendo em vista os achados desse estudo, infere-se que, mesmo a comunicação sendo considerada ferramenta imprescindível para a relação humana e fazendo parte da prática diária dos enfermeiros na Atenção Básica à Saúde, ela ainda é pouco visualizada como uma comunicação de notícias difíceis. Para os estudantes, as instituições formadoras 
necessitam avançar frente à atribuição de comunicar notícias difíceis na Atenção Básica á Saúde, o que fica evidenciado ao relatarem que o termo notícia difícil os remete a casos mais graves, possivelmente envolvendo a possibilidade de morte associada mais ao ambiente hospitalar do que a rede básica, o que pode ser explicado pela formação voltada para o meio hospitalar.

Além disso, compreendem que postergam a comunicação com os usuários, principalmente por falta de preparo. A falta de preparo está intimamente relacionada à falta de abordagem na graduação, o que dificulta o reconhecimento e atuação dos profissionais, apresentando assim fragilidade no currículo do curso de graduação enfermagem e na sua desenvoltura profissional.

\section{REFERÊNCIAS}

1. Pereira BC, Freitas LA, Gonçalves AM, Santos RP, Vilela SC. Interpersonal communication and its implication in nursing. Cult. cuid. [internet], 2019 [cited 2019 Aug 8]; 23(53):230-38. DOI: https://doi.org/10.14198/cuid.2019.53.22.

2. Bastos RA, Fonseca ACG, Pereira AKS, Silva e Souza LC. Health Care Professionals' Training in Communicating Bad News in Oncologic Palliative Care. Rev. bras. cancerol. [internet], 2016 [cited 2019 Aug 8]; 62(3): 263-66. Available From: http://www1.inca.gov.br/rbc/n_62/v03/pdf/10-artigo-opiniao-formacao-dos-profissionais-de-saude-na-comunicacao-de-masnoticias-em-cuidados-paliativos-oncologicos.pdf.

3. Silva AE, Sousa PA, Ribeiro RF. Communication of bad news: perception of physicians working in oncology. Rev. enferm. Cent.Oeste Min. [internet], 2018 [cited 2019 Aug 8]; 8:e2482. DOI: https://doi.org/10.19175/recom.v8i0.2482.

4. Bumb M, Keefe J, Miller L, Overcash J. Breaking Bad News: an evidence-based review of communication models for oncology nurses. Clin. j. oncol. nurs. [internet], 2017 [cited 2019 Aug 8]; 21(5):573-80. DOI: https://doi.org/10.1188/17.CJON.573-580.

5. Vinuto JA. A amostragem em bola de neve na pesquisa qualitativa: um debate em aberto. Temáticas - Revista de PósGraduandos em Ciências Sociais da Unicamp. [internet], 2014 [cited 2019 Aug 8]; 22(44):203-20. Available From: https://www.ifch.unicamp.br/ojs/index.php/tematicas/article/view/2144/1637.

6. Busanello J, Lunardi Filho WD, Kerber NPC, Santos SSC, Lunardi VL, Pohlmann FC. The focus group as a technique for data collection. Cogitare enferm. [internet], 2013 [cited 2019 Jun 24]; 18(2):358-64. DOI: http://dx.doi.org/10.5380/ce.v18i2.32586.

7. Moraes R, Galiazzi MC. Análise Textual Discursiva. 2 ed. Editora: Unijuí. 2013.

8. Koch CL, Rosa AB, Bedin SC. Bad news: meanings attributed in neonatal/pediatric care practices. Rev. bioét. (Impr.). [internet], 2017 [cited 2019 Sep 18]; 25(3):577-84. DOI: http://dx.doi.org/10.1590/1983-80422017253214.

9. Campos CACA, Silva LB, Bernardes JS, Soares ALC, Ferreira SMS. Challenges of communication in Neonatal Intensive Care Unit for professionals and users. Saúde debate. [internet], 2017 [cited 2019 Aug 8]; 41(esp.):165-74. DOI: https://doi.org/10.1590/0103-11042017S214.

10. Fontes $\mathrm{CMB}$, Menezes DV, Borgato $\mathrm{MH}$, Luiz MR. Communicating bad news: an integrative review of the nursing literature. Rev. bras. enferm. [internet], 2017 [cited 2019 Sep 19]; 70(5):1089-95. DOI: https://doi.org/10.1590/0034-7167-2016-0143.

11. Karnieli-Miller O, Neufeld-Kroszynski G. The potential of argumentation theory in enhancing patient-centered care in breaking bad news encounters. Journal of Argumentation in Context, [internet], 2018 [cited 2019 Sep 22]; 7(2):120-37. DOI: https://doi.org/10.1075/jaic.18023.kar.

12. Silveira FJF, Botelho CC, Valadão CC. Breaking bad news: doctors' skills in communicating with patients. São Paulo med. j. [internet], 2017 [cited 2019 Sep 19]; 135(4): 323-31. DOI: http://dx.doi.org/10.1590/1516-3180.20160221270117.

13. Ferreira SRS, Périco LA, Dias VRFD. The complexity of the work of nurses in Primary Health Care. Rev. bras. enferm. [internet], 2018 [cited 2019 Sep 19]; 71(1):704-9. DOI: http://dx.doi.org/10.1590/0034-7167-2017-0471.

14. Santos RCA, Miranda FAN. Importance of the bond between profesional and user in family health strategy. Rev. enferm. UFSM. [internet], 2016 [cited 2019 Sep 19]; 6(3):350-9. DOI: http://dx.doi.org/10.5902/2179769217313.

15. Brasil. Ministério da Saúde. Secretaria de Atenção à Saúde. Departamento de Atenção Básica. Caderno de Atenção Básica. Acolhimento a demanda espontânea - queixas mais comuns na Atenção Básica [internet] 2013. [cited 2021 Mai 3]; 2(28):236237. Available From: http://bvsms.saude.gov.br/bvs/publicacoes/acolhimento_demanda_espontanea_queixas_comuns_cab28v2.pdf.

16. Seifart C, Hofmann M, Bär T, Riera Knorrenschild J, Seifart U, Rief W. Breaking bad news-what patients want and what they get: evaluating the Spikes protocol in Germany. Ann Oncol. [internet], 2014 [cited 2021 Mai 4]; 25(3):707-11. DOI: http://dx.doi.org/10.1093/annonc/mdt582.

17. Baile WF, Buckman R, Lenzi R, Glober G, Beale EA, Kudelka AP. Spikes: a six-step protocol for delivering bad news: application to the patient with cancer. Oncologist. [internet], 2000 [cited 2021 Mai 4]; 5(4):302-11. DOI: http://dx.doi.org/10.1634/theoncologist.5-4-302.

18. Silva LPS, Santos I, Castro SZM. Giving bad news in the context of cancer care: integrative literature review. Rev. enferm. UERJ. [internet], 2016 [cited 2019 Sep 19]; 24(3):1-8. DOI: http://dx.doi.org/10.12957/reuerj.2016.19940.

19. Rocha L, Melo C, Costa R, Anders JC. The Communication of bad news by nurses in the context of obstetric care. REME rev. min. enferm. [internet], 2016 [cited 2019 Sep 18]; 20:e981. DOI: https://doi.org/10.5935/1415-2762.20160051.

20. Newman AR. Nurses' perceptions of diagnosis and prognosis-related communication: An Integrative Review. Cancer nurs. [internet], 2016 [cited 2019 Sep 19]; 39(5):E48-E60. DOI: https://doi.org/10.1097/NCC.0000000000000365. 